\title{
ENANISMO Y PODREDUMBRE BASAL DE Eustoma grandiflorum Y SU RELACIÓN CON LA DENSIDAD DE Fusarium solani EN EL SUELO
}

\author{
SILVIA M. WOLCAN ${ }^{1,2,3}$, GLADYS A. LORI ${ }^{1,2,3}$, LIA RONCO ${ }^{1,2}$, ADRIÁN \\ F. MITIDIERI ${ }^{1}$ \& ROBERTO FERNÁNDEZ ${ }^{4}$
}

${ }^{1}$ Facultad de Ciencias Agrarias y Forestales, Universidad Nacional de La Plata, calle 60 y 119, c.c. 31,1900 La Plata,
Buenos Aires, Argentina, fax: 542214252346 , e-mail: swolcan@interar.com.ar; ${ }^{2}$ Centro de Investigaciones de
Fitopatología; ${ }^{3}$ Comisión de Investigaciones Científicas de la Provincia de Buenos Aires; ${ }^{4}$ Instituto
Nacional de Tecnología Agropecuaria (INTA) Gran Buenos Aires

(Aceptado para publicación en 22/06/2001)

Autor para correspondencia: Silvia M. Wolcan

WOLCAN, S.M., LORI, G.A., RONCO, L., MITIDIERI, A.F. \& FERNÁNDEZ, R. Enanismo y podredumbre basal de Eustoma grandiflorum y su relación con la densidad de Fusarium solani en el suelo. Fitopatología Brasileira 26:710-714. 2001.

\section{RESUMEN}

En cultivos comerciales de lisianthus de La Plata y alrededores (Argentina), se observó que Fusarium solani afecta al $100 \%$ de los establecimientos productivos provocando enanismo y podredumbre basal. Se realizó un ensayo para analizar la densidad de $F$. solani del suelo bajo el efecto de distintos tratamientos y relacionarla con la evolución de ambas patologías. Los análisis del suelo se hicieron en 3 épocas: antes del transplante (octubre), al inicio (enero) y al final (marzo) de la floración. En enero y marzo se evaluó la incidencia de cada enfermedad y se cuantificaron las plantas cosechadas. La población de $F$. solani no varió en las 3 épocas y se redujo significativamente con los fumigantes y el vapor. Esto determinó una incidencia del enanismo (enero) de 0 a $9,5 \%$ con los primeros y de $31,4 \%$ con el vapor. Para el testigo y los fungicidas la incidencia varió entre el 87,9 y el $100 \%$, disminuyendo las plantas cosechadas. La podredumbre basal comenzó con el ingreso del patógeno a través de las heridas producidas por la cosecha. Ésta fue significativamente mayor con los fumigantes (90,7 a $99 \%$ ) y también con el vapor $(76,8 \%)$, produciéndose la mayor incidencia en marzo $(15,7$ a 22,2 y $10,1 \%$ respectivamente), reduciéndose el período productivo. Se destaca la importancia de disminuir la densidad de $F$. solani en el momento del transplante. Entre los métodos de control el Metam sodio y Dazomet podrían ser eficaces sustitutos del Bromuro de metilo, seguidos por el vapor.

Palabras clave adicionais: lisianthus, población de Fusarium solani, incidencia, alternativas al Bromuro de metilo.

\section{ABSTRACT \\ Stunt and basal rot of Eustoma grandiflorum and its relationship with Fusarium solani density in the soil}

Fusarium solani was recorded in $100 \%$ of the commercial crops of lisianthus (Eustoma grandiflorum) located around La Plata (Argentina). The pathogen causes stunt and basal rot. A trial was carried out to analyse the $F$. solani soil density and its relation to the development of both diseases. Soil samples were analysed at three times: before transplant (October), at the beginning of flowering (January) and at the ending of flowering (March) of flowering. The incidence of each disease was evaluated in January and March, and the plants harvested were quantified. The soil density of F. solani was not altered during the three times for each treatment, and fumigants and steam decrease it significantly. In January the stunt incidence reached 0 to $9.5 \%$ with the fumigants and $31.4 \%$ with the steam treatment. In the control and fungicide plots, the incidence reached 87.9 to $100 \%$, and a consequent reduction of flower production was observed. Stem rot began after the flowers were harvested as a result of pathogen entry through the wounds. The major values of harvested plants were obtained with fumigants (90.7 to $99 \%$ ) and steam $(76.8 \%)$, so the greatest stem rot incidence was 15.7 to 22.2 and $10.1 \%$, respectively, in March, and the productive period was shortened. The need of reducing $F$. solani soil density before lisianthus transplant is emphasized. Among soil control treatments, Metham sodium and Dazomet could be effective substitutes for Methil bromide followed by steam treatment.

\section{INTRODUCCION}

El lisianthus [Eustoma grandiflorum (Raf.) Shinn.] es un cultivo que se destina para flor de corte y producción en macetas. En los últimos años se ha incrementado la superficie cultivada en la provincia de Buenos Aires (zona núcleo de cultivo) debido a la mayor difusión de esta flor y al incremento de su comercialización en el país y en el exterior 
(Fernández, 2000).

Dentro de las enfermedades fúngicas que lo afectan, se destacan las ocasionadas por Fusarium spp.: Fusarium solani (Mart.) Sacc. provocando podredumbre del tallo y damping off (Taubenhaus \& Ezekiel, 1934) y recientemente enanismo (Wolcan et al., 2001), F. oxysporum Schlecht. f. sp. eustomae causando marchitamiento (Raabe, 1985) y F. avenaceum (Fr.: Fr.) Sacc. ocasionando podredumbre del tallo y de la corona (Koike et al., 1996). De las especies mencionadas, en la Argentina sólo se observó la presencia de $F$. solani que provoca importantes pérdidas de la producción a causa de la podredumbre basal (Wolcan y Lori, 1996) y el enanismo (Wolcan et al., 2001).

Los objetivos del trabajo fueron evaluar la evolución del enanismo y de la podredumbre basal y relacionar la densidad de $F$. solani en el suelo con su actividad patógena, a lo largo del ciclo del cultivo bajo distintas condiciones de control.

\section{MATERIALES Y METODOS}

\section{Relevamiento de las enfermedades}

Durante los últimos cuatro años se realizó un relevamiento en 23 invernáculos cultivados con lisianthus, ubicados en 12 establecimientos localizados en el área de producción florícola de La Plata y alrededores. Se hicieron observaciones en los distintos estados de desarrollo del cultivo y se extrajeron muestras de plantas enfermas y de suelo para practicar aislamientos en el laboratorio.

\section{Evolución de las enfermedades y control del patógeno}

En la campaña 1998/99 se condujo un ensayo en un invernáculo destinado a la producción comercial de lisianthus. Como consecuencia del monocultivo, el suelo estaba altamente infestado con $F$. solani. Se empleó el cultivar Echo 1 cuya sanidad fue previamente comprobada mediante aislamientos en agar de papa glucosado (APG) a partir de ejemplares tomados al azar.

A efectos de evaluar la evolución de las enfermedades y su relación con la población de $F$. solani en el suelo, se consideraron distintos parámetros durante los cinco meses del ensayo (octubre a marzo), que coincidieron con la etapa productiva del cultivo. Asimismo se analizó el efecto de distintos tratamientos de suelo sobre la población de $F$. solani. Se probaron tres fumigantes, vapor de agua y dos fungicidas indicados para el control de Fusarium spp. Los fungicidas se incluyeron con la finalidad de comprobar su efecto sobre el patógeno en su habitat natural y en ausencia del cultivo, evaluando la consecuente respuesta del mismo. Los tratamientos consistieron en: Testigo; $60 \mathrm{~g} / \mathrm{m}^{2}$ de Bromuro de metilo, líquido fumigante $98 \% ; 70 \mathrm{~g} / \mathrm{m}^{2}$ de Dazomet, granulado $98 \% ; 125 \mathrm{cc} / \mathrm{m}^{2}$ de Metam Sodio, líquido floable 32\%; Vapor de agua; $4 \mathrm{cc} / \mathrm{m}^{2}$ de Carbendazim, suspensión concentrada $50 \%$ y $1,5 \mathrm{cc} / \mathrm{m}^{2}$ de Procloraz, emulsión concentrada $45 \%$.

Se trabajó con parcelas de 2,5 m de largo, distribuídas en cuatro canteros de $0,90 \mathrm{~m}$ de ancho por $18 \mathrm{~m}$ de largo y elevados a $10 \mathrm{~cm}$ de los pasillos. Para no superponer el efecto de tratamientos contiguos, se consideró parcela experimental el centro de esos sectores, abarcando seis hileras con seis plantas por hilera.

La densidad de $F$. solani en el suelo se analizó en tres épocas: inmediatamente después de la aplicación de los productos y antes del transplante (octubre), al comenzar la cosecha de flores (enero) y próximo a la finalización de la cosecha (marzo). De cada parcela se tomó una muestra compuesta por diez submuestras extraídas a una profundidad de hasta $15 \mathrm{~cm}$. Se aplicó el método de las diluciones y el medio selectivo de Nash \& Snyder (1962) modificado (Lori \& Wolcan, 1996) seleccionando previamente la dilución de $10^{-2}$. La densidad de $F$. solani se expresó en unidades formadoras de colonias por gramo de suelo seco (ufc/g).

En enero y marzo se evaluaron la Incidencia y la Severidad de las enfermedades dentro de cada parcela.

La Incidencia de la enfermedad (IE) se calculó según la fórmula:

$$
\text { IE }(\%)=\frac{\left.\mathrm{N}^{o} \text { de plantas enfermas (enanismo o podredumbrebasal }\right)}{\mathrm{N}^{\circ} \text { total de plantas }(\text { sanas }+ \text { enfermas })} \times 100
$$

Para calcular la Severidad de la enfermedad (SE) se categorizaron las plantas con la ayuda de una escala visual de daño con valores de 0 a 3 . En el caso del enanismo la escala consistió en: $0=$ planta sana; $1=$ desarrollo anormal de las plantas, con aspecto intermedio entre los valores 0 y 2 de la escala; 2 = planta con síntomas de detención de crecimiento ( 3 a $8 \mathrm{~cm}$ de altura, tallos delgados, hojas verde pálido - amarillento) y $3=$ planta muerta. Para la podredumbre basal $0=$ planta sana; $1=$ comienzo de plasmólisis de las hojas; 2 = hojas superiores plasmolizadas, de color verde grisáceas con curvatura del ápice de las plantas y hojas basales amarillas y $3=$ planta muerta. Los valores 1 y 2 se observan principalmente en los rebrotes de la corona después del corte de la rama floral.

Con estos valores se determinó la SE en función del número de plantas enfermas de la parcela, aplicando la fórmula:

$$
\mathrm{SE}=\frac{(\mathrm{n} \times 1)+(\mathrm{n} \times 2)+(\mathrm{n} \times 3)}{\mathrm{N}^{\mathrm{o}} \text { de plantas enfermas }}
$$

Donde $\mathrm{n}=\mathrm{N}^{\circ}$ de plantas afectadas por cada uno de los valores de la escala $(1,2$ y 3$)$.

Finalmente en cada parcela se evaluó el porcentaje de plantas cosechadas, tomando en cuenta las plantas con valor 0 de la escala, que tuvieron por lo menos un corte de flor hasta la finalización del ensayo.

A lo largo del ciclo, dentro de cada tratamiento se recolectaron plantas con síntomas, extraídas en las proximidades del sector central evaluado, a los efectos de confirmar la etiología en estudio.

El diseño experimental fue un factorial en bloques al azar con tres repeticiones, donde el factor A estuvo constituído por los meses de observación (épocas) y el factor B por los métodos de control (tratamientos). Previo al análisis de varianza los datos fueron transformados a $\log _{10} n+1$ para las ufc y en arco seno y raíz cuadrada para la incidencia, severidad 
y plantas cosechadas. Para la comparación de medias se empleó el test de Duncan $(\mathrm{P} \leq 0,05)$. Se correlacionaron las densidades de $F$. solani y las distintas variables evaluadas para ambas enfermedades $(\mathrm{P} \leq 0,01)$. Para el análisis de los datos se utilizó el programa estadístico Mstat 2,10.

\section{RESULTADOS Y DISCUSIÓN}

\section{Relevamiento de las enfermedades}

En el $100 \%$ de los establecimientos se detectó la presencia de $F$. solani asociado a plantas con enanismo y con podredumbre basal, encontrándose tanto en suelos previamente cultivados con esta especie, como en aquellos implantados por primera vez. Ambas enfermedades fueron más frecuentes y severas en los suelos que no tuvieron tratamiento previo de control, siendo el Bromuro de metilo el tratamiento más utilizado.

F. solani se aisló a partir del $84 \%$ de las plantas con enanismo y del $99 \%$ de plantas con podredumbre basal. La densidad de $F$. solani en el suelo de las rizosferas de las plantas adultas sanas varió entre 750 y 31.400 ufc/g y entre 20.250 y $64.000 \mathrm{ufc} / \mathrm{g}$ en las rizosferas de las plantas adultas enfermas.

\section{Evolución de las enfermedades y control del patógeno}

En la Tabla 1 se transcriben los valores promedio de las densidad de $F$. solani, la incidencia y la severidad de ambas enfermedades dentro de cada época (factor A) y de cada tratamiento (factor B). Para la densidad de F. solani no hubo interacción entre ambos factores y no se halló diferencia significativa entre las tres épocas. Por otra parte se observó una correlación medianamente alta $(r=0,66,0,62$ y 0,72$)$ entre las densidades de octubre - enero, octubre - marzo y enero - marzo respectivamente. Si bien el ensayo se condujo en un suelo altamente infestado como consecuencia del monocultivo, tal como se observó para $F$. solani en otros hospedantes (Burke \& Kraft, 1974), los análisis realizados indicaron que a lo largo del período evaluado (cinco meses), aún ante la presencia de las plantas de lisianthus la densidad del patógeno se mantuvo estable, sin haberse inducido la recolonización del suelo. Entre los tratamientos de control (Tabla 1), los fumigantes y el vapor redujeron significativamente la densidad de $F$. solani sin que se observara diferencia entre ellos, mientras que los fungicidas no difirieron con el testigo. La eficacia del Metam sodio y del Dazomet para el control de Fusarium fue comprobada para F. oxysporum Schlecht. f. sp. dianthi (Prill. \& Del.) Snyder. \& Hans. (Semer, 1987; García et al., 1995). Con relación a los fungicidas, Gamboa \& Larroque (1985/86) probaron el efecto del Carbendazim y del Prochloraz sobre $F$. oxysporum f. sp. dianthi en aplicaciones previas al transplante. Los resultados obtenidos con el primero fueron semejantes a los del presente ensayo, dado que no redujeron la densidad de los patógenos en el suelo. El Prochloraz, en cambio, fue efectivo para el control de $F$. oxysporum f. sp. dianthi provocando el retraso en la aparición de la enfermedad (Gamboa \& Larroque, 1985/86). En el presente trabajo los dos fungicidas resultaron ineficaces para reducir la densidad de $F$. solani en el suelo (Tabla 1 y Figura 1A) y al continuar en el ensayo se comportaron como el tratamiento testigo. En las Figura 1 se observa el comportamiento de la población de $F$. solani frente a los distintos tratamientos durante el mes de octubre (Figura 1A) y su efecto sobre la cantidad de plantas cosechadas (Figura 1B). En la Figura 2 se grafican las épocas de mayor incidencia del enanismo (Figura 2A) y de la podredumbre (Figura 2B) frente a cada tratamiento. Una elevada densidad de $F$. solani en mes de octubre (Figura 1A) determinó posteriormente una alta incidencia de enanismo (Figura 2A) y en consecuencia un bajo porcentaje de plantas

TABLA 1 - Resultados de las variables evaluadas durante el ciclo del cultivo de lisianthus (Eustoma grandiflorum)

\begin{tabular}{|c|c|c|c|c|c|}
\hline \multirow{2}{*}{$\begin{array}{l}\text { Factores } \\
\qquad \text { Época }\end{array}$} & \multirow{2}{*}{$\begin{array}{l}\text { Densidad de } F \text { : } \\
\text { solani (ufc/g) }\end{array}$} & \multicolumn{2}{|c|}{ Enanismo $^{2}$} & \multicolumn{2}{|c|}{ Podredumbre basal ${ }^{3}$} \\
\hline & & Incidencia $(\%)$ & Severidad & Incidencia \%) & Severidad \\
\hline Octubre & $5,83 \mathrm{a}^{4}$ & - & - & - & - \\
\hline Enero & $9,45 \mathrm{a}$ & $45,66 \mathrm{a}$ & $1,45 \mathrm{a}$ & - & - \\
\hline Marzo & $8,12 \mathrm{a}$ & 34,27 b & $1,24 \mathrm{a}$ & - & - \\
\hline $\begin{array}{c}\text { Tratamiento } \\
\text { (B) }\end{array}$ & & & & $\begin{array}{l}\text { (A x } \\
\text { Mar } \\
\end{array}$ & \\
\hline Bromuro de metilo & $0,55 \quad \mathrm{c}$ & $0,009 \quad \mathrm{e}$ & $3,00 \mathrm{a}$ & $15,70 \mathrm{a}$ & $3,00 \mathrm{a}$ \\
\hline Metam sodio & $4,56 \mathrm{bc}$ & $2,75 \quad \mathrm{~d}$ & $0,55 \mathrm{~b}$ & $16,63 \mathrm{a}$ & $2,35 \mathrm{a}$ \\
\hline Dazomet & $2,22 \quad \mathrm{c}$ & $8,20 \quad d$ & $1,70 \mathrm{ab}$ & $16,20 \mathrm{a}$ & $1,83 \mathrm{ab}$ \\
\hline Vapor & $1,33 \mathrm{c}$ & $33,70 \quad c$ & $0,88 \quad b$ & $10,10 \mathrm{a}$ & $2,80 \mathrm{a}$ \\
\hline Carbendazim & $15,00 \mathrm{a}$ & $91,20 \mathrm{a}$ & $1,87 \mathrm{ab}$ & $0,00 \mathrm{~b}$ & $0,00 \quad b$ \\
\hline Prochloraz & $14,55 \mathrm{a}$ & $88,86 \mathrm{ab}$ & $1,85 \mathrm{ab}$ & $0,90 \mathrm{~b}$ & $1,00 \quad b$ \\
\hline Testigo & $15,22 \mathrm{a}$ & $73,56 \quad b$ & $1,60 \mathrm{ab}$ & $0,90 \mathrm{~b}$ & $1,00 \mathrm{~b}$ \\
\hline \multicolumn{6}{|c|}{$\begin{array}{l}{ }^{1} \text { Multiplicar x } 10^{2} \text {. } \\
{ }^{2} \text { No hubo interacción entre los factores A y B. } \\
{ }^{3} \text { Hubo interacción entre A y B. }\end{array}$} \\
\hline
\end{tabular}




\section{A Población de Fusarium solani (octubre)}

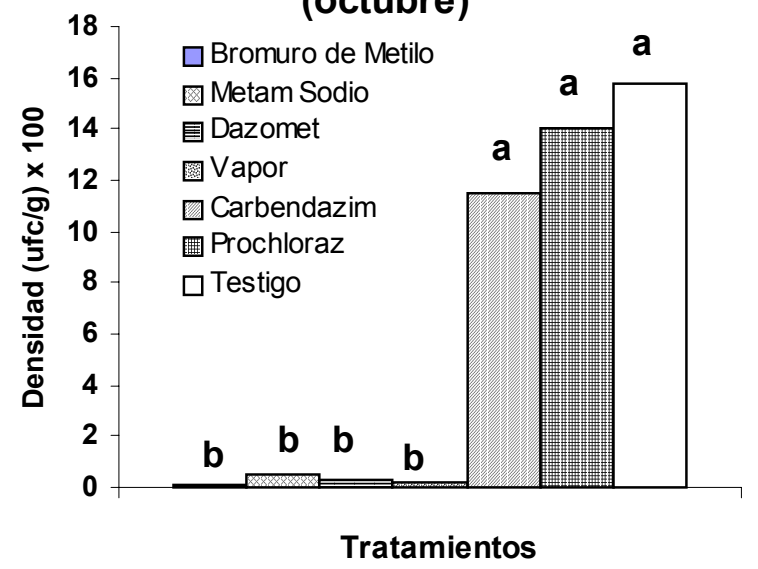

B Plantas cosechadas

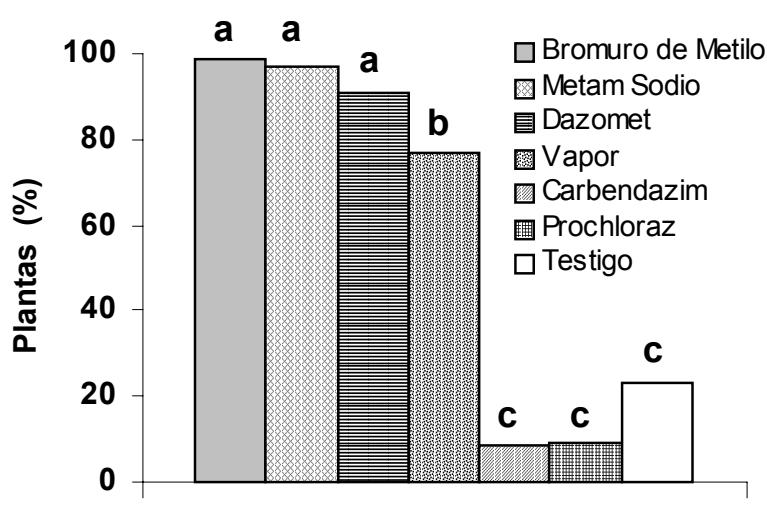

Tratamientos

FIG. 1 - Densidad de Fusarium solani del suelo previo al transplante de lisianthus (Eustoma grandiflorum) (A) y su relación con la cantidad de plantas cosechadas (B), para los distintos tratamientos. Las mismas letras sobre las barras indican que no hubo diferencia significativa $(P \leq 0,05)$ según el Test de Duncan.

cosechadas (Tabla 1 y Figura 1B). Además, se establecieron correlaciones medianamente altas entre la densidad de $F$. solani en octubre y la incidencia del enanismo en enero y marzo ( $r=0,71$ y 0,68 respectivamente). Esto podría indicar que la cantidad de inóculo en el suelo en el momento del transplante, tendría un efecto directo sobre la manifestación posterior de la enfermedad. Una respuesta similar fue obtenida por Scherm et al., (1998) estudiando la enfermedad denominada muerte súbita de la soja (Glycine max L.) ocasionada por $F$. solani (Mart.) Sacc. f. sp. glycines. Al buscar relaciones entre variables biológicas, físicas y químicas del suelo con la severidad de la enfermedad, encontraron que la población de $F$. solani fue la variable que mostró la correlación más elevada sobre la severidad.

Con relación al enanismo no hubo interacción entre las épocas y los tratamientos evaluados. Al considerar las épocas (Tabla 1), la incidencia se redujo significativamente en el mes de marzo, debido a que, si bien luego de algunas semanas muchas de las plantas afectadas mantuvieron los síntomas iniciales y otras murieron, predominaron aquellas que evolucionaron hasta alcanzar un desarrollo normal. Los tres fumigantes redujeron significativamente la incidencia, existiendo una diferencia muy notoria con el testigo y los fungicidas, mientras que el vapor alcanzó un resultado intermedio. La severidad no mostró diferencia significativa entre las dos épocas evaluadas. Con relación a los
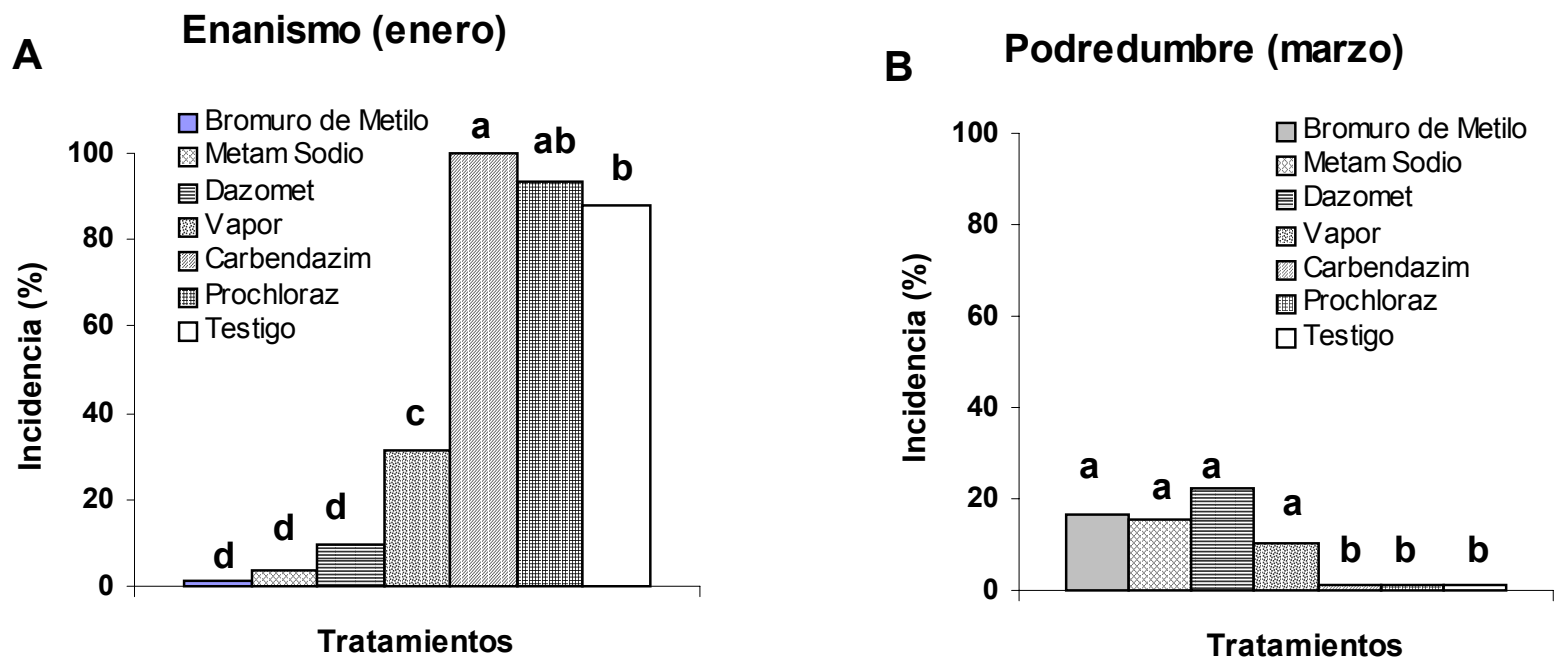

FIG. 2 - Incidencia de las enfermedades causadas por Fusarium solani en lisianthus, (Eustoma grandiflorum) de acuerdo con su evolución dentro del ciclo del cultivo, para cada tratamiento: (A) enanismo y (B) podredumbre basal. Las mismas letras sobre las barras indican que no hubo diferencia significativa $(P \leq 0,05)$ según el Test de Duncan. 
tratamientos, los valores más altos, que fueron inferiores al 2 de la escala, se observaron indistintamente en aquellos muy o nada eficaces para el control de la enfermedad (Tabla 1).

En el análisis estadístico de la podredumbre basal (Tabla 1) se halló interacción entre las épocas y los tratamientos (A x B). En enero no se registraron plantas con esta enfermedad que sí se manifestó en la evaluación de marzo (Figura 2B). La incidencia de la podredumbre basal fue significativamente mayor en los tratamientos que redujeron la incidencia del enanismo (fumigantes y vapor) y que presentaron mayor cantidad de plantas cosechadas (Tabla 1, Figuras $1 \mathrm{~B}$ y $2 \mathrm{~A}$ y B). Para el testigo y los fungicidas la incidencia de la podredumbre fue casi nula, dadas las pocas plantas que llegaron a la cosecha, hallándose una correlación $(r=0,71)$ entre la incidencia de esta enfermedad y las plantas cosechadas. Con la severidad (valores entre 2 y 3 de la escala) se obtuvieron resultados similares a los de la incidencia frente a los tratamientos (Tabla 1).

Al analizar la evolución de las enfermedades, en el ensayo se observó que $F$. solani ocasiona inicialmente el enanismo, que determina que las plantas sean improductivas o que lleguen tardíamente a la cosecha con flores de baja calidad. En el manejo comercial del cultivo de lisianthus, es habitual el tratamiento del suelo antes de la plantación. Como consecuencia de esta práctica, no se observa una incidencia tan elevada de enanismo y esta enfermedad puede pasar inadvertida, ya que los síntomas pueden confundirse con problemas de arraigue de los plantines (Wolcan et al., 2001). Luego de la cosecha se presenta la podredumbre basal, dado que la herida producida por el corte de la flor permite el ingreso del patógeno, más aún cuando el corte se realiza a ras del suelo. El tocón afectado ya no rebrota o produce nuevos tallos que se marchitan en distintos estados de crecimiento, acortando el período productivo y haciendo que el cultivo se deba abandonar anticipadamente.

Se destaca la importancia de la densidad de F. solani en el suelo en el momento de la implantación del cultivo, por lo que se hace necesario reducir la cantidad de inóculo, por ejemplo mediante el uso de tratamientos de control. Si bien el fumigante empleado comúnmente es el Bromuro de metilo, de acuerdo con el Protocolo de Montreal, debido a la contaminación ambiental que provoca (reducción de la capa de ozono, contaminación de napas, etc.) se impone su sustitución gradual y progresiva en el mundo. Los resultados del presente trabajo demostraron que el Dazomet y el Metam sodio, seguidos por el Vapor serían eficaces sustitutos del Bromuro de metilo para el control de $F$. solani en el cultivo de lisianthus.

\section{AGRADECIMIENTOS}

El trabajo fue realizado dentro del marco del Proyecto "Alternativas al uso del bromuro de metilo en frutilla, tomate y flores de corte" (MP/ARG/97/186), ONUDI - OPROZ. Agradecemos a la Ing. Agr. Jorgelina Rolleri y a la Lic. Cecilia Mónaco por su participación en las tareas de Laboratorio y al Ing. Agr. Antonio Kitagawa y al Sr. productor Seiji Kiwata por su colaboración en las tareas de campo.

\section{REFERENCIAS BIBLIOGRÁFICAS}

BOOTH, C. The genus Fusarium. Commonwealth Mycological Institute, Kew, Surrey, England. 1971.

BURKE, D.W. \& KRAFT, J.M. Responses of beans and peas to root pathogens accumulated during monoculture of each crop species. Phytopathology 64:546-549. 1974.

FERNÁNDEZ, R. El lisianthus una experiencia productiva. INTA Informa 60:6-7. 2000.

GAMBOA, B.S. \& LARROQUE, O.R. Ensayos "in vitro" y de campo para el control de Fusarium oxysporum f. sp. dianthi (Prill. y Del.) Snyd. y Hans. Revista de la Facultad de Agronomía 61 y 62:153-158. 1985/6.

GARCÍA, D.L., ARBELÁEZ, M.G. \& ARBELÁEZ G.T. Tratamiento físico y químico del suelo para el control del marchitamiento vascular del clavel causado por el hongo Fusarium oxysporum f. sp. dianthi. Agronomía Colombiana XII:8-20. 1995.

KOIKE, S.T., GORDON, T.R. \& LINDOW, S.E. Crown rot of Eustoma caused by Fusarium avenaceum in California. Plant Disease 80:1429. 1996.

LORI, G.A. \& WOLCAN, S.M. Fusarium spp. del suelo. Identificación mediante observación en placa del medio de Nash \& Snyder modificado. Revista Iberoamericana de Micología 13:18-23. 1996.

NASH, S.M. \& SNYDER, W.C. Quantitative estimations by plate counts of propagules of the bean root rot Fusarium in field soil. Phytopathology 52:567-572. 1962.

RAABE, R.D. Fusarium wilt of Eustoma grandiflora. Phytopathology 75:1306. 1985. (Abstract).

SCHERM, H., YANARG, X.B. \& LUNDEEN, P. Soil variables associated with Sudden Death Syndrome in soybean fields in Iowa. Plant Disease 82:1152-1157. 1998.

SEMER, C.R. Basamid and Methyl bromide compounds as fumigants in carnation and chrysanthemum production in selected propagation media. Proceedings of Florida State of Horticultural Society 100:330-334. 1987.

TAUBENHAUS, J.J. \& EZEKIEL, W.N. Two new diseases of the Texas bluebell, Eustoma russellianus. Phytopathology 24:19. 1934. (Abstract).

WOLCAN, S.M. \& LORI, G.A. Podredumbre basal de Eustoma grandiflora en la Argentina:Etiología. Investigación Agraria: Producción y Protección Vegetales 11:465-472. 1996.

WOLCAN, S.M., LORI, G. \& RONCO, L. First report of Fusarium solani causing stunt on Lisianthus. Plant disease 85:443. 2001. 